\title{
Limited Streamer Tube System for Detecting Contamination in the Gas Used in the BABAR Instrumented Flux Return
}

\author{
Laura Ingalls Huntley \\ SLAC-TN-06-024 \\ Office of Science, SULI Program \\ Franklin \& Marshall College \\ Stanford Linear Accelerator Center \\ Menlo Park, California
}

August 25, 2006

Prepared in partial fulfillment of the requirements of the Office of Science, U.S. Department of Energy Science Undergraduate Laboratory Internship (SULI) Program under the direction of Dr. Mark Convery in the BABAR group at the Stanford Linear Accelerator Center.

Participant:

Signature

Research Advisor:

$$
\text { Signature }
$$




\section{Table of Contents}

$\begin{array}{ll}\text { Abstract } & 3\end{array}$

$\begin{array}{ll}\text { Introduction } & 4\end{array}$

Materials and Methods $\quad 5$

$\begin{array}{ll}\text { Results } & 7\end{array}$

$\begin{array}{lr}\text { Discussion and Conclusions } & 8\end{array}$

$\begin{array}{ll}\text { Acknowledgements } & 9\end{array}$

$\begin{array}{lr}\text { References } & 9\end{array}$

$\begin{array}{ll}\text { Tables } & 10\end{array}$

$\begin{array}{ll}\text { Figures } & 11\end{array}$ 


\begin{abstract}
Limited Streamer Tube System for Detecting Contamination in the Gas Used by the BABAR Instrumented Flux Return. High Energy Physics, Office of Science, SULI Program, Stanford Linear Accelerator Center, Summer 2006. LAURA HUNTLEY (Franklin \& Marshall College, Lancaster, PA 17603) MARK CONVERY (Stanford Linear Accelerator Center, Menlo Park, CA 94025).
\end{abstract}

The Resistive Plate Chambers (RPCs) initially installed in the Instrumented Flux Return (IFR) of the BABAR particle detector have proven unreliable and inefficient for detecting muons and neutral hadrons. In the summer of 2004, the BABAR Collaboration began replacing the RPCs with Limited Streamer Tubes (LSTs). LST operation requires a mixture of very pure gases and an operating voltage of $5500 \mathrm{~V}$ to achieve maximum efficiency. In the past, the gas supplies obtained by the BABAR Collaboration have contained contaminants that caused the efficiency of the IFR LSTs to drop from approximately 90\% to approximately 60\%. Therefore, it was necessary to develop a method for testing this gas for contaminants. An LST test system was designed and built using two existing LSTs, one placed $1 \mathrm{~cm}$ above the other. These LSTs detect cosmic muons in place of particles created during the BABAR experiment. The effect of gas contamination was mimicked by reducing the operating voltage of the test system in order to lower the detection efficiency. When contaminated gas was simulated, the coincidence rate and the percent coincidence between the LSTs in the test system dropped off significantly, demonstrating that test system can be used as an indicator of gas purity. In the fall of 2006, the LST test system will be installed in the gas storage area near the BABAR facility for the purpose of testing the gas being sent to the IFR. 


\section{INTRODUCTION}

The BABAR Collaboration at the Stanford Linear Accelerator Center endeavors to measure fundamental differences between matter and anti-matter through the study of electronpositron collisions at high energy. These collisions are designed to create pairs of B and anti-B mesons, which in turn decay into other hadrons and leptons. By measuring the lifetimes of the B mesons and identifying their decay products, BABAR physicists are able to study charge parity violation, as well as many other interesting physical phenomena [1].

The outermost component of the BABAR particle detector is an Instrumented Flux Return (IFR), which shapes the magnetic field as well as provides identification for muons and neutral hadrons produced by the electron-position collisions. The IFR consists of layers of particle detectors fitted between layers of steel [2]. Muons, which only interact electromagnetically with matter, are able to travel through more of the steel layers than other particles, making it possible to identify them [3]. Originally, the detectors in the IFR were a new, cost-effective technology called Resistive Plate Chambers (RPCs). RPCs consist of parallel plastic plates at high voltage (HV). When a particle passes through the gas mixture between these plates, ionization occurs, and a streamer signal is produced [4]. Since irregularities in the surfaces of the plates can also cause electron flow to occur, the plates are coated with linseed oil in order to keep them smooth. Unfortunately, the efficiency of the BABAR RPCs has decreased rapidly, most likely due to defects in the plate surfaces and contaminants in the linseed oil, and they have become unreliable for detecting particles. As attempts made to repair them were unsuccessful, two of the six RPC sectors were replaced with Limited Streamer Tubes (LSTs) in the summer of 2004. The remaining four sectors will be installed in the fall of 2006 (See Figure 1). 
The LSTs contain long cells filled with a gas composed of 3\% argon, $8 \%$ isobutane, and 89\% carbon dioxide by volume [5]. When charged particles enter the LSTs, they ionize the gas, causing a small number of free electrons to be produced. These electrons drift toward the HV wires running through the tubes. If the voltage is large enough, a cascade of electrons is created, causing an electronic response in the $\mathrm{HV}$ wires. However, if the voltage is excessive, secondary cascades will be produced, causing false signals. With pure gas and operating at the optimal voltage of 5500V, the LSTs are approximately 90\% efficient at detecting particles. The gas used in the LSTs is bought by SLAC from an outside source and mixed on-site at a gas storage area near the BABAR facility. In the past, the isobutane supplies have contained impurities that caused the detection efficiency of the IFR to drop to approximately 60\%. It was determined that, in order to prevent efficiency loss in the future, a method for testing the purity of the gas was needed [6].

In the summer of 2006, we designed and assembled an LST test system for the purpose of detecting contaminated gas before it enters the BABAR IFR. This system detects cosmic muons, which pass through horizontal surfaces at an approximate rate of $1 \mathrm{~cm}^{-2} \mathrm{~min}^{-1}$ [7], in place of particles produced by electron-positron collisions. The accompanying electronics make it possible to measure the coincidence rate, or the number of detections made by both LSTs per unit time, and the percent coincidence, or the ratio of coincidental detections to the number of detection made by the top LST. As the number of coincidental detections is proportional to the product of the efficiencies of the LSTs, both the rate and percent of coincidence are very sensitive to decreased efficiency in the LSTs and are thus good indicators of contaminated gas. 


\section{MATERIALS AND METHODS}

Two LST modules, one resting $1 \mathrm{~cm}$ above the other and each measuring $50.5 \mathrm{~cm}$ x 15.4 $\mathrm{cm}$ x $2.0 \mathrm{~cm}$, were used to create the gas testing system (See Figure 2). The LSTs are configured such that gas may be pumped through the LSTs in series and out through a bubbler. The gas

used during our test was bought pre-mixed from an off-site company. Because it was impractical to pollute this gas for testing purposes, impure gas was simulated by decreasing the voltage in the top LST below the optimum range.

Aside from being significantly shorter, the LSTs in the testing system are identical to those used in the BABAR IFR. Each LST consists of eight long cells, which are coated with a graphite paint kept at ground potential. Each cell contains a gold-plated wire at variable HV. These wires are paired into four input-output channels on the end of the LST (See Figure 3). A HV connector box plugs into each of these channels as well as a ground connection. The frontend electronics are housed in an Ortec Nuclear Instruments \& Methods (NIM) Bin, Model 401B (See Figure 4). HV from a Bertan HV Power Supply, Model 1739P, is fed into the connector box and travels through a $1 \mathrm{M} \Omega$ resistor to the LST. If a particle triggers an electronic cascade, the analog signal travels back into the connector box and through a $1 \mathrm{nF}$ capacitor to the frontend electronics (See Figure 5).

The front-end electronics take signals from the LSTs and use NIM modules to measure the number of muons passing through the LSTs. A LeCroy Octal Discriminator, Model 623B, receives the signals from the connector box and outputs a fixed-width pulse for every signal above the threshold voltage, which we set at approximately $30 \mathrm{mV}$. A LeCroy 4-Fold Logic Unit, Model 365AL, uses OR gates to bundle the channels of each LST together. The signals 
from the OR gates are then sent to a LeCroy Dual Gate Generator, Model 222, which output square-wave pulses of width $10 \mu$ s. The signal from the top LST then goes to a Joerger Visual Scalar, Model VS, where the number of pulses is counted. It is also sent, along with the signal from the bottom LST, to a LeCroy Quad Coincidence Gate, Model 622, where an AND gate sends a pulse to another counter in the Visual Scalar if the two LSTs detect a muon at the same time. Figure 6 shows a block diagram of the front-end electronics.

The coincidence rate can be measured by counting the number of coincidental detections in a known period of time. The percent coincidence can be found by dividing the coincidental counts by the number of detections made by the top LST. In order to increase the stability and magnitude of the coincidence count, only the inner two channels in the top LST were used, as they are more likely to detect muons that will also pass through the bottom LST.

\section{RESULTS}

We measured the average number of cosmic muons passing through the top LST to be $(1.261 \pm .059) \mathrm{cm}^{-2} \mathrm{~min}^{-1}$ and the bottom LST to be $(1.229 \pm .022) \mathrm{cm}^{-2} \mathrm{~min}^{-1}$. These flux measurements agree with literature value of approximately $1 \mathrm{~cm}^{-2} \mathrm{~min}^{-1}$ [7] and demonstrate that our LSTs are functioning properly.

The number of muons a single LST detects per some length of time as a function of the voltage supplied to the LST is called a "singles rate test." For this test, we varied the voltage from $4800 \mathrm{~V}$ to $5500 \mathrm{~V}$ and recorded the number of detections in 10 seconds. Functional LSTs usually show an edge on this curve at $5300 \mathrm{~V}$ and a plateau around $5500 \mathrm{~V}$ [5]. We found that our LSTs meet these expectations (see Figure 7). 
With pure gas and with both LSTs operating at the optimal voltage of $5500 \mathrm{~V}$, we measured a $(241.6 \pm 6.5) \mathrm{min}^{-1}$ coincidence rate and $(46.6 \pm 1.1) \%$ coincidence. Table 1 shows the changes in coincidence rate and percent coincidence over a period of several hours.

Figures 8 and 9 show the coincidence rate and the percent coincidence as a function of the voltage in the top LST while the bottom LST is held at $5500 \mathrm{~V}$. We conducted these tests by varying the voltage in the top LST from $4800 \mathrm{~V}$ to $5500 \mathrm{~V}$ and measuring the number of coincidental detections and the number of detections made by the top LST during a period of five minutes. As can be seen in the Figures, both the coincidence rate and the percent coincidence increase as the voltage, and therefore the efficiency, of the top LST approaches the optimal voltage range and then decrease as the excessive voltage begins to create false signals.

\section{DISCUSSION AND CONCLUSIONS}

We conclude that our LSTs presently meet performance expectations on the evidence of their measurements of muon flux and the singles rate test. In the future, however, it will be necessary to repeat these tests on a case-by-case basis in order to determine whether any efficiency losses are actually the result of impure gas and not due to LST malfunction.

As can been seen from Figures 8 and 9, both the coincidence rate and the percent coincidence are sensitive to changes in LST efficiency. The coincidence rate test has the advantage of not depending on the detections made by a single LST module, which is important since x-rays can sometimes be detected by one LST and cause the percent coincidence to drop despite the fact that no efficiency losses have occurred. On the other hand, the percent coincidence is a simpler test to perform, as the coincidence rate test requires the use of a 
stopwatch or timer. Furthermore, while the coincidence rate is theoretically more reliable, Table 1 shows that the percent coincidence did not vary significantly over a period of several days.

We recommend that both tests be used according to the needs and time-constraints of the test system operator. For both tests, measurements should be made for at least five minutes in order to obtain sufficiently accurate and precise results. The test system will be installed as an intermediary between the gas storage area and the main BABAR detector in the fall of 2006, where it will be used to check gas being sent to the BABAR IFR for contamination

\section{ACKNOWLEDGEMENTS}

This research was conducted at the Stanford Linear Accelerator Center. I thank the U. S. Department of Energy, Office of Science for providing with the opportunity to participate in the exciting research conducted at SLAC. I also thank my mentor, Mark Convery, for his guidance and advice. Finally, I thank the coordinators and participants of the Science Undergraduate Laboratory Internships program at SLAC for their effort, dedication, and friendship.

\section{REFERENCES}

[1] P.F. Harrison and H.R. Quinn, Eds., The BABAR Physics Book, Menlo Park, California: Stanford Linear Accelerator Center, 1998.

[2] B. Aubert, et al., "The BABAR Detector," Nuclear Instruments and Methods in Physics Research A, vol. 479, pp.1-116, 2002.

[3] J. Perl, et al., "The BABAR Detector," [Online document], May 2006, [cited 2006 Aug. 11], Available at HTTP: http://www.slac.stanford.edu/BFROOT/www/doc/workbook /detector/detector.html. 
[4] R. Santonico and R. Cardarelli, "Development of Resistive Plate Counter,” Nuclear Instruments and Methods, vol. 187, pp. 377-380, 1981.

[5] W. Menges, “The BABAR Muon System Upgrade,” Nuclear Science Symposium Conference Record, 2005 IEEE, vol. 3, pp. 1470-74.

[6] M. Convery, "LST Operations and Installation Plans," presented at the BABAR Collaboration Meeting, Montreal, QC, 2006.

[7] W.-M. Yao, et al., "2006 Review of Particle Physics,” Journal of Physics G, vol. 33, no. 1, Jul., pp. 1-1232.

\section{TABLES}

\begin{tabular}{|l|l|l|}
\hline Time & Coincidence Rate $\left(\mathrm{min}^{-1}\right)$ & Percent Coincidence (\%) \\
\hline \hline 0 days 0 hrs. & $242.8 \pm 7.0$ & $44.96 \pm .96$ \\
0 days 1.5 hrs. & $250.4 \pm 7.1$ & $47.39 \pm .97$ \\
5 days 3 hrs. & $235.0 \pm 6.9$ & $46.37 \pm .99$ \\
5 days 4 hrs. & $250.2 \pm 7.1$ & $48.34 \pm .98$ \\
6 days 4 hrs. & $233.2 \pm 6.8$ & $45.82 \pm .99$ \\
6 days 5 hrs. & $238.4 \pm 6.9$ & $46.06 \pm .98$ \\
7 days 4.5 hrs. & $238.6 \pm 6.9$ & $46.17 \pm .98$ \\
7 days 6 hrs. & $244.2 \pm 7.0$ & $47.64 \pm .99$ \\
\hline
\end{tabular}

Table 1. Coincidence rate and percent coincidence taken at different times over a period of several days. The mean of the coincidence rate is $(241.6 \pm 6.5) \mathrm{min}^{-1}$ and the mean of the percent coincidence is $(46.6 \pm 1.1) \%$. 


\section{FIGURES}

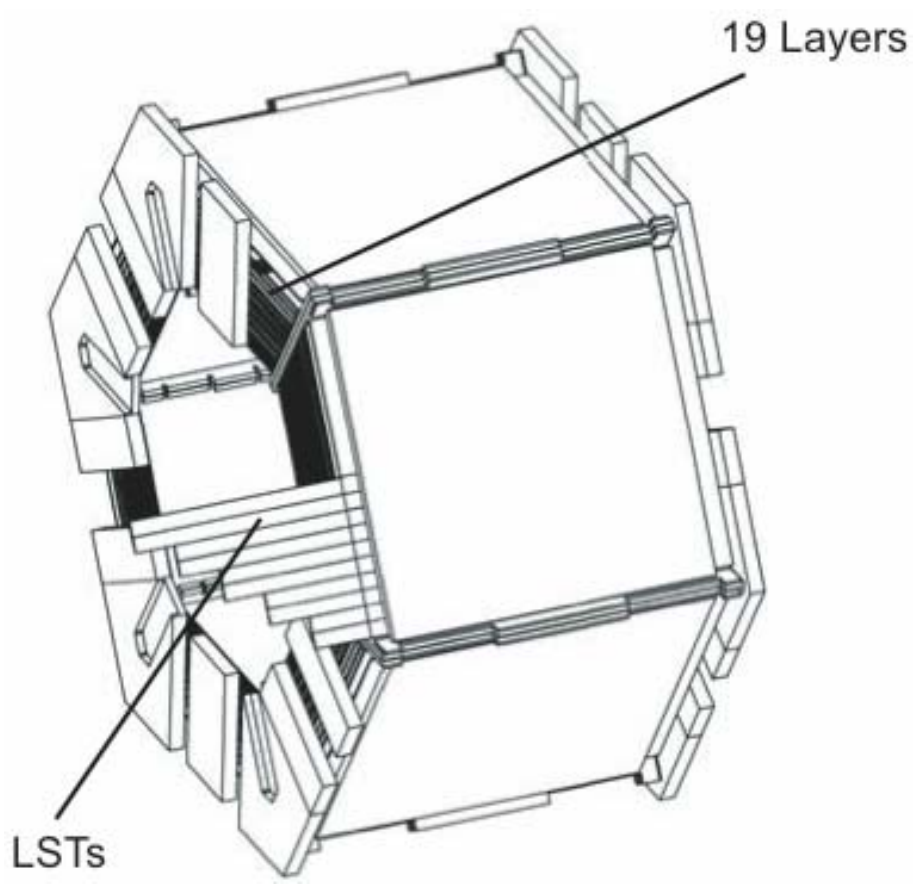

Figure 1. LSTs being inserted into the gaps between the steel layers of the BABAR IFR.

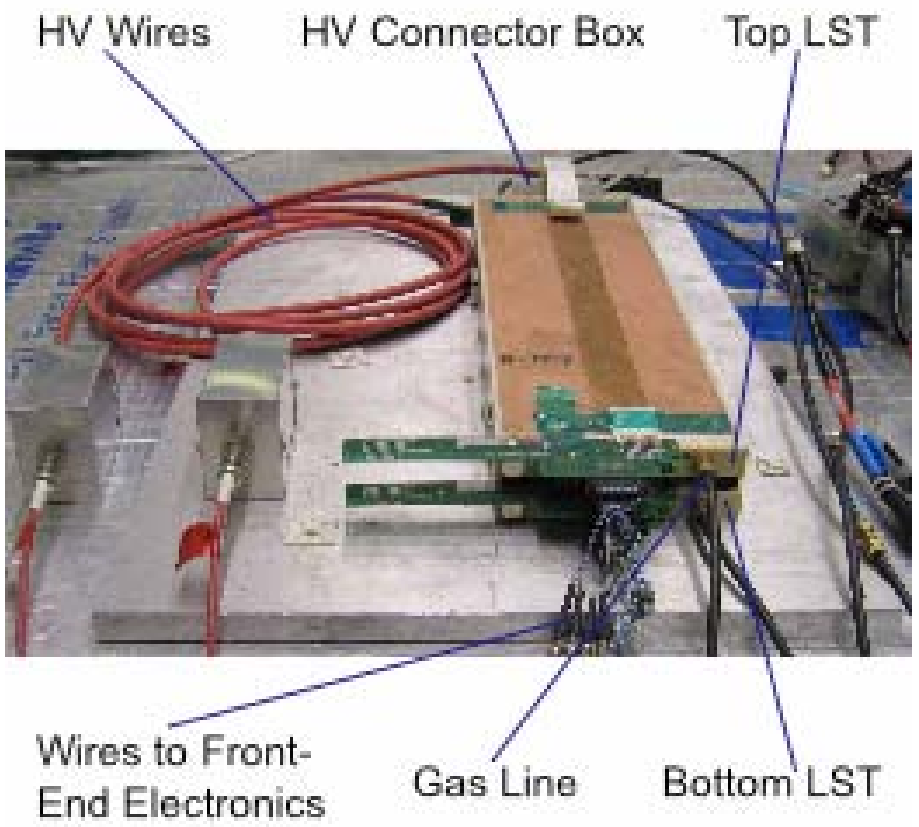

Figure 2. LST test system consisting of two LSTs, one placed $1 \mathrm{~cm}$ above the other. 


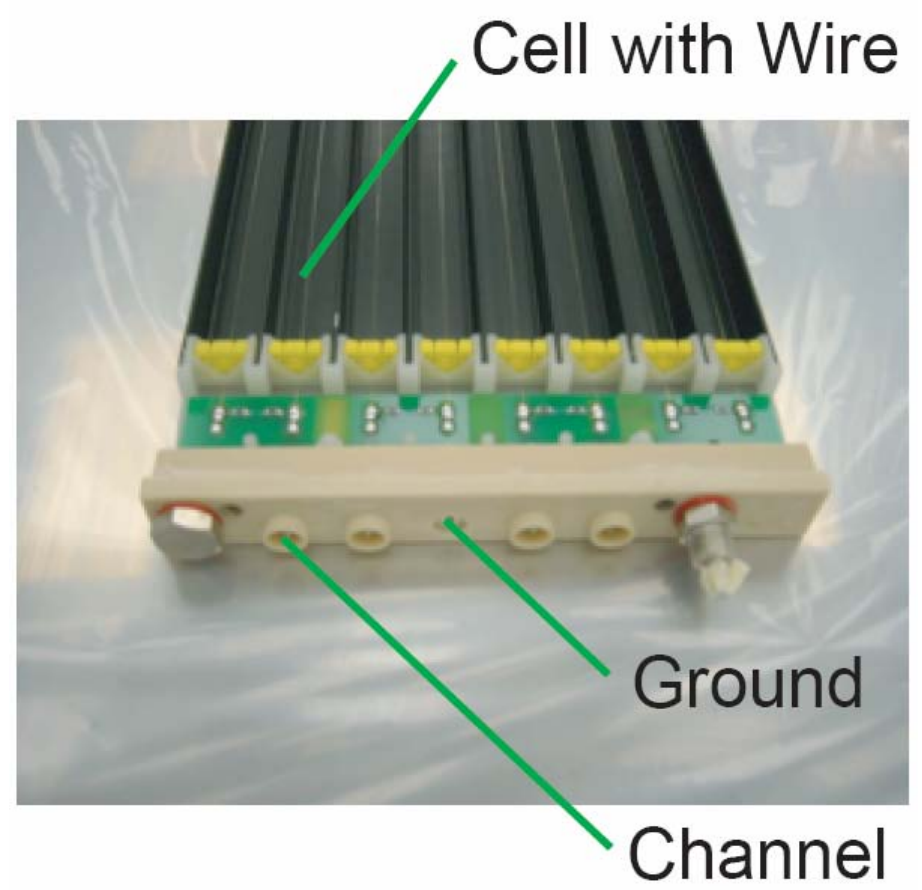

Figure 3. LST shown without plastic sleeve. Eight cells coated in a graphite-based paint contain one gold-plated wire each. Two wires comprise one channel. The end-cap of the LST has plugs for four channels, along with a ground pin.

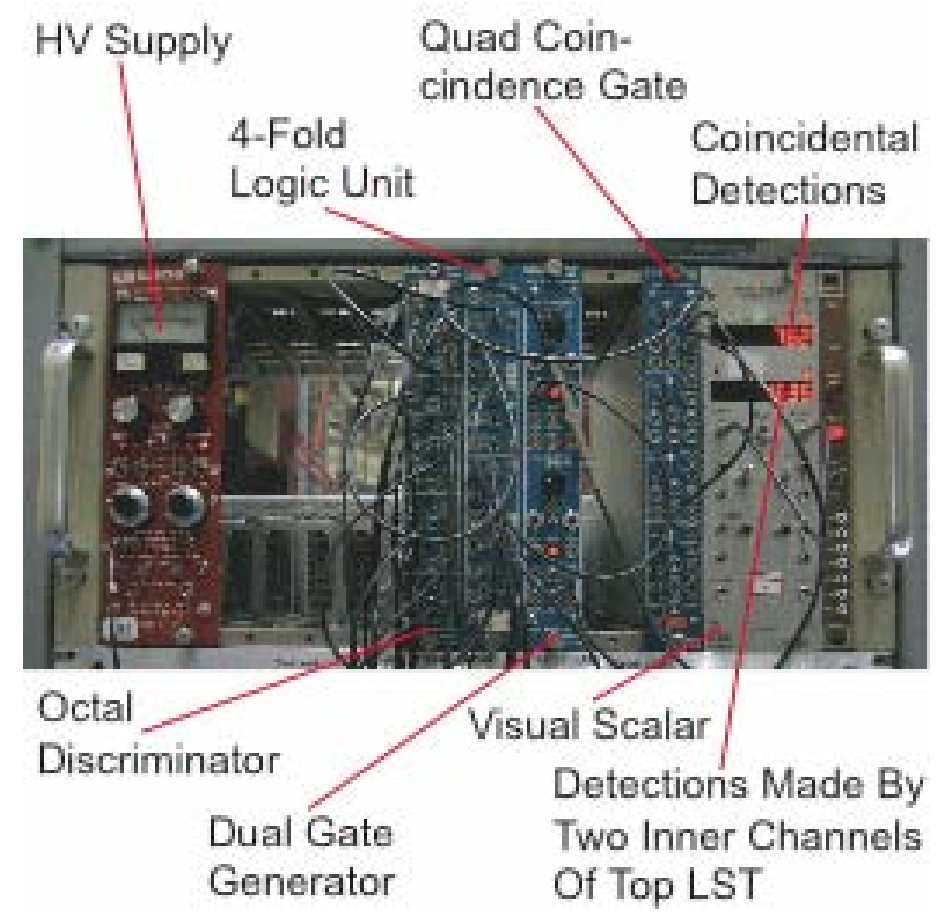

Figure 4. Ortec NIM Bin, Model 401B, with HV power supply and front-end electronics. 

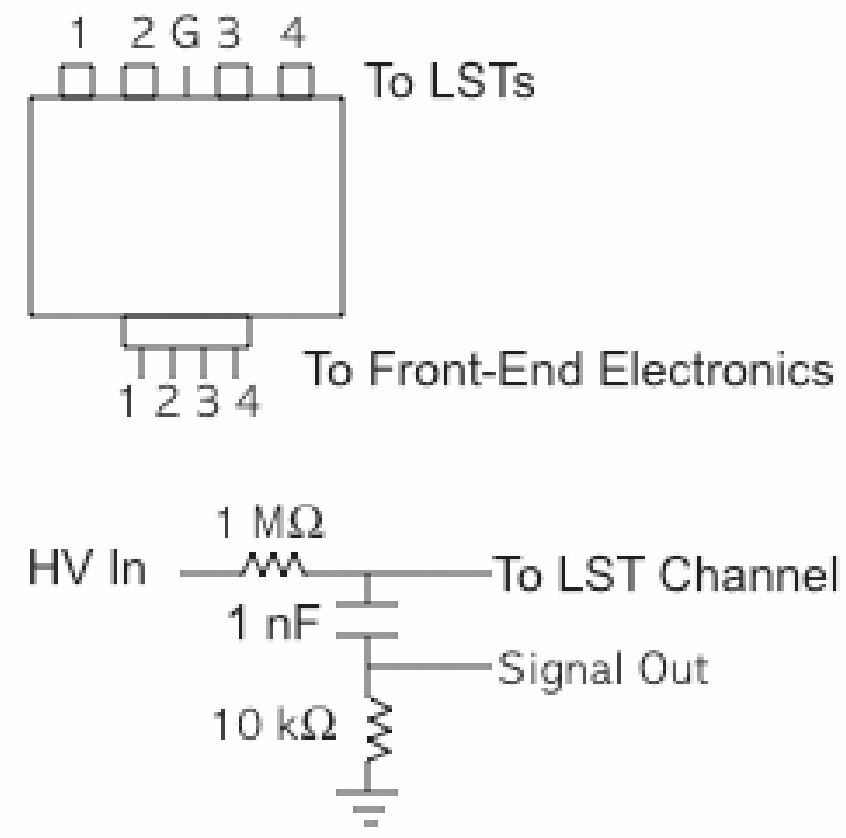

Figure 5. HV Connector Box. The top diagram shows connector box with four channels and a ground plug, plus four output wires to the front-end electronics. The bottom diagram shows the circuitry for one channel in the connector box.

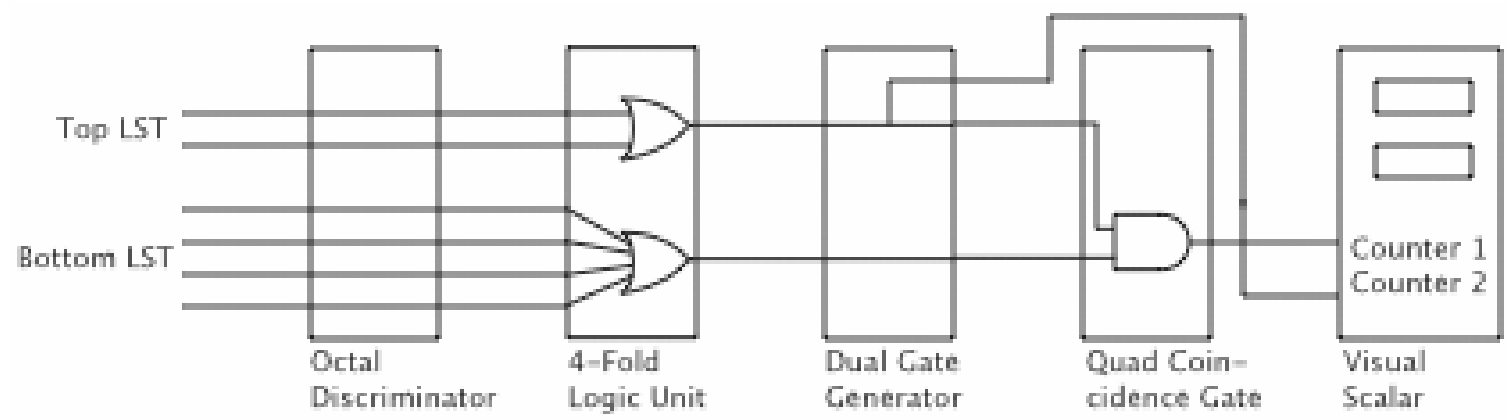

Figure 6. Block diagram of front-end electronics. Counter 1 displays the number of detections made coincidentally by both LSTs and Counter 2 displays the number of detections made by the inner two channels of the top LST. 


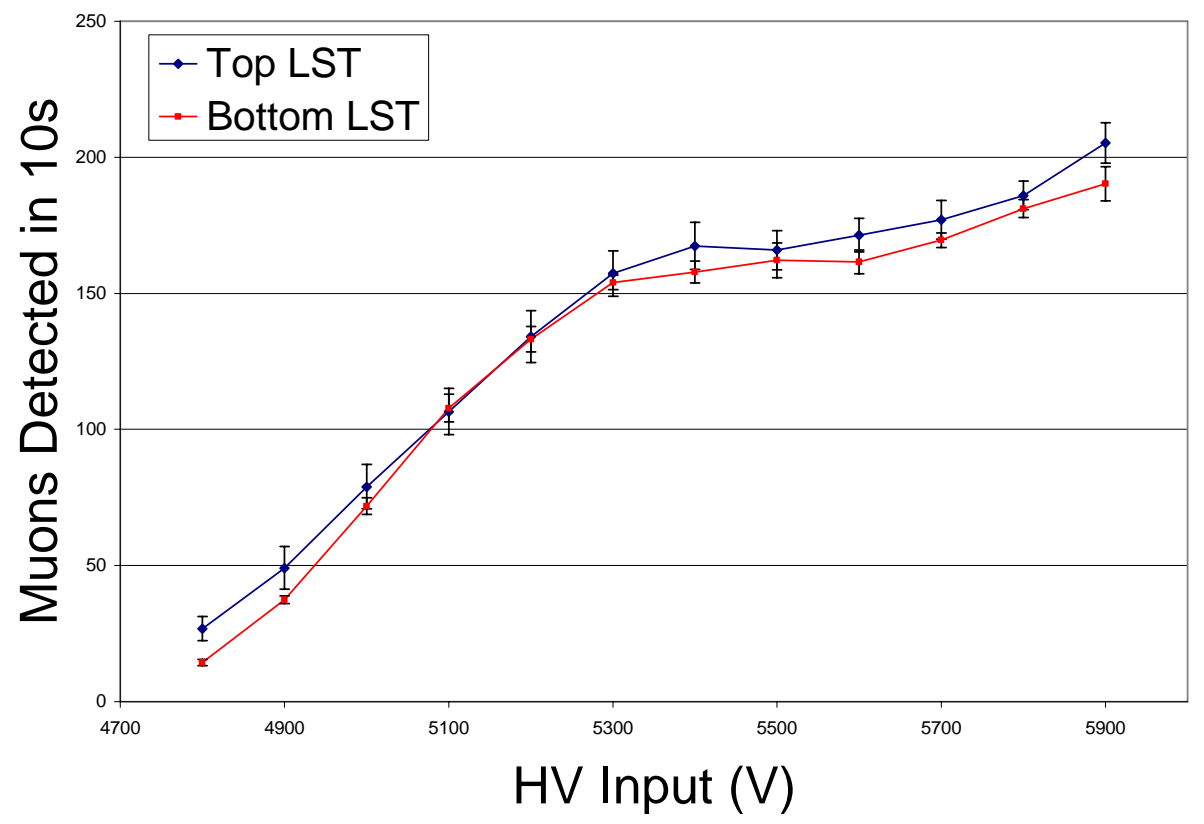

Figure 7. Singles rate tests for the top and bottom LSTs. Both curves show an edge at approximately $5300 \mathrm{~V}$ and plateau around $5500 \mathrm{~V}$.

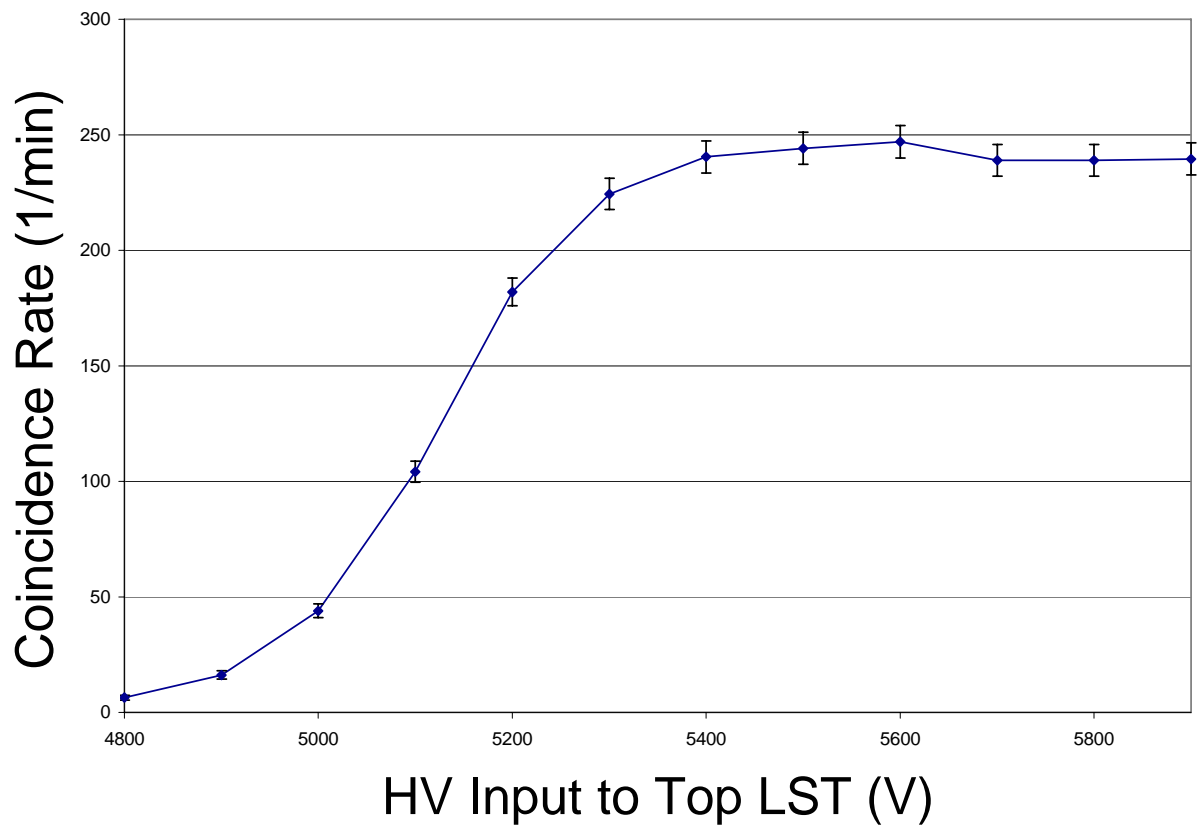

Figure 8. Coincidence rate as a function of the voltage supplied to the top LST as the bottom LST is help at optimal operating voltage, $5500 \mathrm{~V}$. 


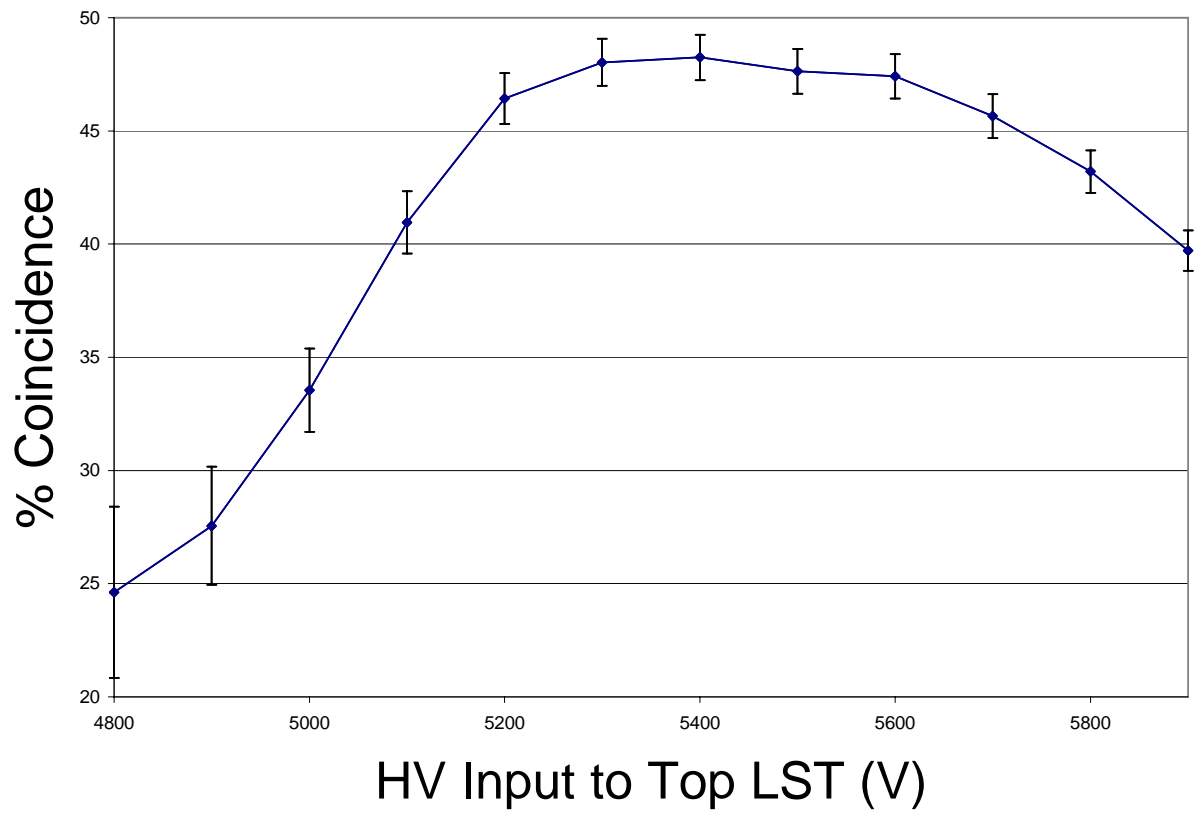

Figure 9. Percent coincidence as a function of the voltage supplied to the top LST as the bottom LST is held at optimal operating voltage, $5500 \mathrm{~V}$. 